\title{
Extreme Points of Polytopes of Discrete Copulas
}

\author{
${ }^{*}$ Elisa Perrone $^{a}$ and Fabrizio Durante ${ }^{b}$ \\ ${ }^{a}$ Department of Mathematics and Computer Science, Eindhoven University of Technology, \\ Groene Loper 3, 5612 AE Eindhoven (The Netherlands) e.perrone@tue.nl \\ ${ }^{b}$ Department of Economic Sciences, Università del Salento, \\ Centro Ecotekne, 73100 Lecce (Italy) fabrizio.durante@unisalento . it
}

\begin{abstract}
Discrete copulas are useful tools in statistics to represent the joint distribution of discrete random vectors. Furthermore, they are fascinating mathematical objects that admit a representation as a convex polytope. In this work, we analyze the set of extreme points of convex polytopes of discrete copulas. We focus on the general class of discrete copulas defined on arbitrary grid domains of the unit square, thereby providing a complete characterization of extremal discrete copulas in the most general setting. To do so, we exploit well-known results on the extreme points of transportation polytopes. Finally, we show that the characterization presented here generalizes previous work on the topic.
\end{abstract}

Keywords: Discrete Copulas, Transportation Polytopes, Extreme points.

\section{Introduction}

Copulas are flexible mathematical tools to obtain marginal-free distributions of multivariate random vectors $[8,10,17]$. Key to the success of copulas in probability and statistics is Sklar's Theorem, which states that the joint distribution function $F_{\mathbf{X}}$ of any $d$ dimensional random vector $\mathbf{X}=\left(X_{1}, \ldots, X_{d}\right)$ can be expressed, for every $\left(x_{1}, \ldots, x_{d}\right) \in \mathbb{R}^{d}$, as

$$
F_{\mathbf{X}}\left(x_{1}, \ldots, x_{d}\right)=C\left(F_{X_{1}}\left(x_{1}\right), \ldots, F_{X_{d}}\left(x_{d}\right)\right),
$$

where the function $C:[0,1]^{d} \rightarrow[0,1]$ is a $d$ dimensional copula and $F_{X_{1}}, \ldots, F_{X_{d}}$ are univariate marginal distributions [25].

We note that the function $C$ in $\mathrm{Eq}(1)$ is uniquely identified on the set $\operatorname{Range}\left(F_{X_{1}}\right) \times \cdots \times \operatorname{Range}\left(F_{X_{d}}\right)$. As a consequence, if $F_{X_{1}}, \ldots, F_{X_{d}}$ are discrete probability distributions with finite support, Sklar's theorem identifies the so-called discrete copulas, which are restrictions of copulas on grid domains of the unit hyper-cube $[12,14,15]$. Discrete copulas can be easily extended to full-domain copulas by spreading the probability mass on each hyper-rectangle of their grid domain, but, in general, the extension is not unique [5]. In spite of that, discrete copulas are useful tools in many applied fields, where practitioners can take advantage of the mathematical properties of these functions alongside their probabilistic interpretation.

One of the most convenient mathematical features of discrete copulas is that they admit a representation as a convex polytope. A convex polytope is a bounded convex space consisting of all points satisfying a finite list of inequalities [27]. Convex polytopes naturally appear in many areas of applied mathematics, including statistics, probability, and operation research. Recent work in dependence modeling shows the several advantages of handling discrete copulas as convex polytopes. As an example, the polytopal representation of discrete copulas has been exploited in hydrology to obtain flexible copula models to generate syntetic data of rainfall totals [19, 20, 22]. Another work shows an application to copula selection in portfolio optimization [13]. In [18], the authors use tools from discrete geometry to define the polytopal representations of subfamilies of discrete copulas with desirable stochastic properties. Such representations can be helpful to design a hypothesis test for a negative dependence property [7].

On the mathematical side, discrete copulas (and discrete quasi-copulas) have been extensively studied in terms of their matrix representations $[1,2,9,12,14$, $15,16,21]$. In [18], the authors highlight that the space of discrete copulas with fixed marginal distributions of finite support of size $p$ and $q$, respectively, corresponds to a polytope known as the generalized Birkhoff polytope [6]. A similar correspondence holds between the space of discrete quasi-copulas and a polytope known 
as the alternating transportation polytope $[11,26]$. The definition of discrete (quasi-) copulas is further generalized in [18] to the discrete (quasi-) copulas on arbitrary grid domains. There, the authors also show a connection between discrete (quasi-) copulas and other classes of (alternating) transportation polytopes.

Besides analyzing the matrix representation of discrete (quasi-) copulas, special attention has been given to the study of those points within the space of discrete (quasi-) copulas that cannot be expressed as a convex combination of other points. These points are the extreme points (or vertices) of the polytope, and they are also known as the irreducible discrete (quasi-) copulas. Notably, knowing the set of all extreme discrete (quasi-) copulas allows to describe any discrete copula as a convex combination of them. This latter aspect can be analogously expressed in the continuous case via Krein-Millman Theorem; see, e.g., [8].

Characterizing the class of the extreme points of convex polytopes is a natural question in discrete geometry. In the context of discrete copulas, such a characterization is well-understood in special cases. For example, in [12], the authors show that the extreme points of the class of $(p \times p)$-discrete copulas are those of minimal range, i.e., the discrete copulas that only take values in the uniform partition of the unit square with step $1 / p$. This is a beautiful result since, as explained in [15], the $(p \times p)$-discrete copulas of minimal range correspond to the so-called empirical copulas, which are the foundation of rank-based (non-parametric) copula approaches [23, 24]. The case of $(p \times q)$-discrete (quasi-) copulas is considered in $[1,2,16]$. There, a first characterization of the set of irreducible discrete copulas when $q=p k$ is also given. Again in this case, the irreducible discrete copulas correspond to the ones with minimal range. The general case $q \neq p k$ appears to be more complex. Recently, in [9], the authors shed more light on the set of irreducible discrete (quasi-) copulas when $q \neq p k$, providing negative answers to two open questions posed in [2].

Despite these results collectively cover many special cases, to the best of our knowledge, a complete characterization of the irreducible discrete copulas is not available in the copula literature. In this paper, we fill this gap and give a characterization of the extreme points of the polytopes of discrete copulas defined on arbitrary grid domains. In particular, we recall a wellknown result in the transportation polytopes literature, thereby showing that the extremality of any discrete copula depends on the zero pattern of its associated transportation matrix.

The remainder of the paper is organized as follows. In Section 2, we introduce basic definitions and known re- sults unifying the theory of discrete copulas and transportation polytopes. In Section 3, we give the characterization of the extreme points. In Section 4, we use such a characterization in several examples of discrete copulas that appear in [2] and [9]. In Section 5, we draw conclusions and briefly discuss the case of discrete quasi-copulas.

\section{Discrete copulas and convex polytopes}

We now present the definition of discrete copulas on non-uniform grid domains, originally introduced in [18], and the associated convex polytopes.

First, we clarify the notation, which is in line with [18]. For $p \in \mathbb{Z}_{>0}$, we denote $[p]=\{1, \ldots, p\},\langle p\rangle=$ $\{0, \ldots, p\}$, and $I_{p}=\{0,1 / p, \ldots,(p-1) / p, 1\}$. Assuming $p$ and $q$ both in $\mathbb{Z}_{>0}$, we consider two vectors $u=\left(u_{1}, \ldots, u_{p}\right) \in \mathbb{R}_{>0}^{p}$ and $v=\left(v_{1}, \ldots, v_{q}\right) \in$ $\mathbb{R}_{>0}^{q}$ with the properties that $u_{p}=v_{q}=p q$, and $u_{i}<u_{i+1}, v_{j}<v_{j+1}$ for $i \in[p-1]$ and $j \in[q-$ 1]. The vectors $u$ and $v$ define two partitions of $[0,1], U_{p}=\left\{0, u_{1} /(p q), \ldots, u_{p} /(p q)\right\}$, and $V_{q}=$ $\left\{0, v_{1} /(p q), \ldots, v_{q} /(p q)\right\}$. As discussed in [18], we can consider discrete copulas $C_{U_{p}, V_{q}}$ defined on $U_{p} \times V_{q}$ as functions which satisfy the properties of a copula on the domain $U_{p} \times V_{q}$.

In particular, the set of all discrete copulas $C_{U_{p}, V_{q}}$ : $U_{p} \times V_{q} \rightarrow[0,1]$, denoted as $\operatorname{DC}\left(U_{p}, V_{q}\right)$, is composed of all matrices $\left(c_{i, j}\right) \in \mathbb{R}^{(p+1) \times(q+1)}$ with $c_{i, j}=$ $C_{U_{p}, V_{q}}\left(u_{i} /(p q), v_{j} /(p q)\right)$ satisfying the following conditions:

(p1) $c_{0, j}=0, c_{i, 0}=0$ with $i \in\langle p\rangle, j \in\langle q\rangle$;

(p2) $c_{p, j}=v_{j} /(p q), c_{i, q}=u_{i} /(p q)$, with $i \in[p], j \in[q]$;

(p3) $c_{i, j}+c_{i-1, j-1}-c_{i-1, j}-c_{i, j-1} \geq 0$ for every $i \in$ $[p], j \in[q]$.

Remark 1. We notice that the definition of discrete copulas on non-uniform grid domains includes the regular case of rectangular discrete copulas defined on $I_{p} \times I_{q}$. Indeed, for $p$ and $q$ in $\mathbb{Z}_{>0}$, we can obtain the uniform rectangular grid domain $I_{p} \times I_{q}$ by choosing $u=(q, 2 q, \ldots, k q, \ldots, p q) \in \mathbb{R}^{p}$ and $v=$ $(p, 2 p, \ldots, k p, \ldots, q p) \in \mathbb{R}^{q}$.

Given two vectors $\tilde{u}=\left(\tilde{u}_{1}, \ldots, \tilde{u}_{p}\right) \in \mathbb{R}_{>0}^{p}$ and $\tilde{v}=$ $\left(\tilde{v}_{1}, \ldots, \tilde{v}_{q}\right) \in \mathbb{R}_{>0}^{q}$, we define the transportation polytope $\mathscr{T}(\tilde{u}, \tilde{v})$ as the convex polytope in the $p q$ variables $x_{i, j}$ satisfying, for all $i \in[p]$ and $j \in[q]$, the following conditions

$$
x_{i, j} \geq 0, \quad \sum_{h=1}^{q} x_{i, h}=\tilde{u}_{i}, \quad \sum_{\ell=1}^{p} x_{\ell, j}=\tilde{v}_{j} .
$$


The vectors $\tilde{u}$ and $\tilde{v}$ are called the margins of $\mathscr{T}(\tilde{u}, \tilde{v})$. In [18], the authors prove a correspondence between the discrete copulas $C_{U_{p}, V_{q}}$ in $\mathrm{DC}\left(U_{p}, V_{q}\right)$ and the matrices within transportation polytopes $\mathscr{T}(\tilde{u}, \tilde{v})$ with homogeneous marginals, i.e., $\sum_{i} \tilde{u}_{i}=\sum_{j} \tilde{v}_{j}=p q$. We report this result below for completeness.

Proposition 1. For a function $C_{U_{p}, V_{q}}: U_{p} \times V_{q} \rightarrow[0,1]$, the following statements are equivalent:

(i) $C_{U_{p}, V_{q}} \in \mathrm{DC}\left(U_{p}, V_{q}\right)$

(ii) There is a $(p \times q)$ transportation matrix $\left(x_{i, j}\right)$ in $\mathscr{T}(\tilde{u}, \tilde{v})$, with $\sum_{h=1}^{q} \tilde{v}_{h}=\sum_{\ell=1}^{p} \tilde{u}_{\ell}=p q$, such that for every $i \in\langle p\rangle, j \in\langle q\rangle$

$$
c_{i, j}=C_{U_{p}, V_{q}}\left(u_{i} /(p q), v_{j} /(p q)\right)=\frac{1}{p q} \sum_{\ell=1}^{i} \sum_{h=1}^{j} x_{\ell, h} .
$$

The linear map $T: \mathbb{R}^{(p+1) \times(q+1)} \longrightarrow \mathbb{R}^{p \times q}$ defined as $T\left(c_{i, j}\right)=c_{i, j}+c_{i-1, j-1}-c_{i, j-1}-c_{i-1, j}$ for all $i \in[p]$ and $j \in[q]$ brings any discrete copula of $\operatorname{DC}\left(U_{p}, V_{q}\right)$ into a transportation matrix in $\{1 /(p q)\} \mathscr{T}(\tilde{u}, \tilde{v})$, with margins given as follows:

$$
\begin{gathered}
\tilde{u}=\left(u_{1}, u_{2}-u_{1}, \ldots, u_{k}-u_{k-1}, \ldots, u_{p}-u_{p-1}\right) \\
\tilde{v}=\left(v_{1}, v_{2}-v_{1}, \ldots, v_{k}-v_{k-1}, \ldots, v_{q}-v_{q-1}\right) .
\end{gathered}
$$

We notice that the scalar factor $1 /(p q)$ does not change the geometric structure of the convex polytope nor its extreme points. Again in this more general case, the transportation matrix associated to a discrete copula represents, up to a scalar factor, the probability mass that lies in each rectangle of the grid domain.

We now give two simple numerical examples to illustrate our general theoretic framework in practice. In particular, the examples show how to move from the space of discrete copulas to the associated transportation polytope, and back.

Example 1. We assume $p=3, q=4$, and we consider the two vectors $u=(2,7,12) \in \mathbb{R}^{3}$ and $v=(3,6,9,12) \in \mathbb{R}^{4}$. Thus, we are working on the grid domain $U_{3} \times V_{4}=\{0,2 / 12,7 / 12,1\} \times$ $\{0,3 / 12,6 / 12,9 / 12,1\}$ represented in Figure 1. The following matrix $C_{1}$ is an example of a discrete copula defined on $U_{3} \times V_{4}$ :

$$
C_{1}=\left(\begin{array}{ccccc}
0 & 0 & 0 & 0 & 0 \\
0 & 2 / 12 & 2 / 12 & 2 / 12 & 2 / 12 \\
0 & 3 / 12 & 6 / 12 & 7 / 12 & 7 / 12 \\
0 & 3 / 12 & 6 / 12 & 9 / 12 & 1
\end{array}\right) .
$$

By applying the standard linear map $T$, we find the associated $(3 \times 4)$ transportation matrix below:

$$
T_{1}=\left(\begin{array}{llll}
2 & 0 & 0 & 0 \\
1 & 3 & 1 & 0 \\
0 & 0 & 2 & 3
\end{array}\right)
$$

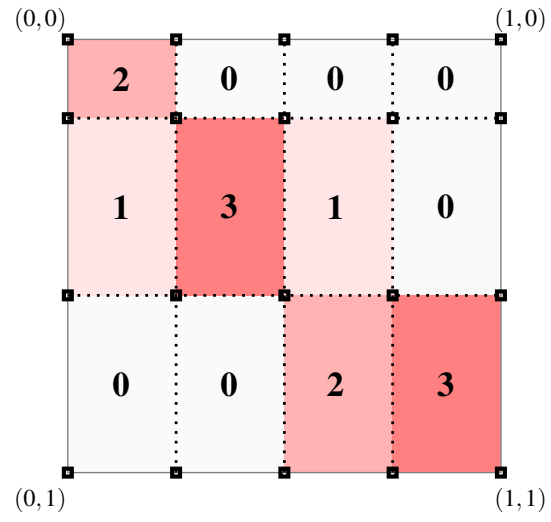

Figure 1: A graphical representation of the grid domain $U_{3} \times V_{4}$ defined in Example 1 alongside the entries of the matrix $T_{1}$ in each corresponding rectangle.

which belongs to the transportation polytope $\mathscr{T}([2,5,5],[3,3,3,3])$, as expected.

Example 2. In this example, we assume $p=q=3$, and we consider the transportation polytope $\mathscr{T}(\tilde{u}, \tilde{v})$, with $\tilde{u}=(1,5,3)$ and $\tilde{v}=(3,2,4)$. We notice that $\sum_{i} \tilde{u}_{i}=\sum_{j} \tilde{v}_{j}=p q=9$. Thus, $\mathscr{T}(\tilde{u}, \tilde{v})$ has homogeneous marginals. We now consider the matrix $T_{2}$ in $\mathscr{T}(\tilde{u}, \tilde{v})$ defined as follows:

$$
T_{2}=\left(\begin{array}{lll}
0 & 1 & 0 \\
1 & 1 & 3 \\
2 & 0 & 1
\end{array}\right)
$$

and we obtain the corresponding discrete copula. To do so, we first find the grid domain $U_{3} \times V_{3}$. By summing up the entries of $\tilde{u}=(1,5,3)$ and $\tilde{v}=(3,2,4)$, we obtain the two vectors $u=(1,6,9)$ and $v=(3,5,9)$. Hence, the grid domain of the discrete copula associated with $T_{2}$ is $U_{3} \times V_{3}=\{0,1 / 9,6 / 9,1\} \times$ $\{0,3 / 9,5 / 9,1\}$, which is represented in Figure 2. From Proposition 1, we can derive the associated discrete copula $C_{2}$, which results in the following matrix:

$$
C_{2}=\left(\begin{array}{cccc}
0 & 0 & 0 & 0 \\
0 & 0 & 1 / 9 & 1 / 9 \\
0 & 1 / 9 & 3 / 9 & 6 / 9 \\
0 & 3 / 9 & 5 / 9 & 1
\end{array}\right)
$$

We now recall a simple way to construct absolutely continuous copulas from discrete copulas.

Remark 2. Any discrete copula can be used to construct an absolutely continuous copula $C:[0,1]^{2} \rightarrow$ $[0,1]$ with piecewise constant density on each rectangle of the partition of $[0,1]^{2}$ determined by $U_{p} \times V_{q}$. The density of a copula $C$ derived by a discrete copula like the ones of Example 1 and Example 2 can be given by $x_{i j}(p q) /\left(\tilde{u}_{i} \tilde{v}_{j}\right)$, where $x_{i j}$ is the entry of the corresponding transportation matrix for $i \in[p]$ and $j \in[q]$. 


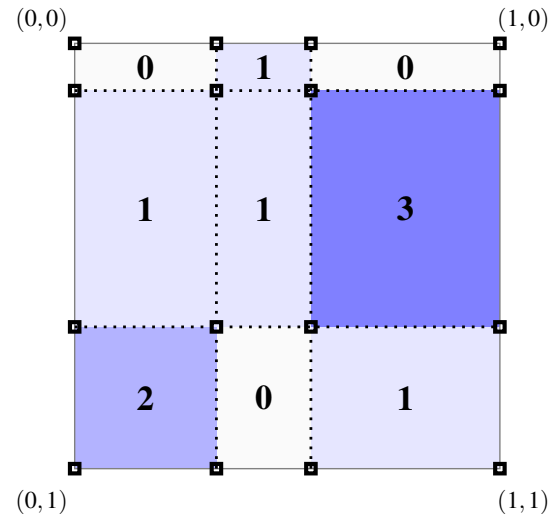

Figure 2: A representation of the grid domain $U_{3} \times$ $V_{3}$ defined in Example 2 alongside the entries of the matrix $T_{2}$ in each corresponding rectangle.

In the next section, we finally present the general characterization of the extreme points of the polytopes of discrete copulas introduced above.

\section{The extreme discrete copulas}

The correspondence between transportation polytopes and discrete copulas allows us to shed light on the set of extreme discrete copulas. Before presenting the details of the characterization, we notice that a similar question, though not involving discrete copulas directly, has been considered in [3] thirty years ago. There, the authors analyze the extreme points of the set of all bivariate distributions with fixed marginals of finite support. In doing so, they draw connections to the transportation polytopes and collect well-known results in the field of discrete geometry, providing five ways of characterizing the (non-) extreme points of their set of distributions. In the following, we only report the statements of the characterization that are useful in our setting. The complete result is given in Theorem 2.9 of [3].

Theorem 1. Let $T_{p, q}$ be any $(p \times q)$-matrix in the transportation polytope $\mathscr{T}(\tilde{u}, \tilde{v})$ with homogeneous marginals, i.e., $\sum_{i} \tilde{u}_{i}=\sum_{j} \tilde{v}_{j}=p q$. Then, the following statements are equivalent:

1. $T_{p, q}$ is not an extreme point of $\mathscr{T}(\tilde{u}, \tilde{v})$.

2. There exists a submatrix $E$ of $T_{p, q}$ such that every row and column of $E$ has at least two positive elements.

3. There exists a square submatrix $D$ of $T_{p, q}$ such that every row and column of $D$ contains at least two positive entries.
4. There exists a square submatrix $F$ of $T_{p, q}$ of order $k \times k$ for some $k \geq 1$ such that the number of positive elements of $F$ is at least $2 k$.

Theorem 1 provides a characterization of (non-) extreme points of transportation polytopes in terms of their zero pattern. A similar characterization for the extreme points of the generalized Birkhoff polytopes has been discussed in [4]. However, the characterization we give in Theorem 1 remains the most general one and the only one that covers the case of discrete copulas defined on arbitrary grid domains introduced in Section 2.

In the next section, we show how to use Theorem 1 to quickly check whether or not a discrete copula is an extreme point, i.e., it is irreducible.

\section{Examples of extreme points}

We now apply Theorem 1 to several examples of irreducible and non-irreducible discrete copulas defined on the uniform grid domain $I_{p} \times I_{q}$ that appear in the literature. We begin with Example 3 in [9], and we discuss the following $(4 \times 6)$ matrix $A_{1}$ (re-scaled according to our normalizing factor):

$$
A_{1}=\left(\begin{array}{llllll}
0 & 2 & 0 & 0 & 2 & 2 \\
2 & 0 & 2 & 2 & 0 & 0 \\
0 & 0 & 2 & 2 & 0 & 2 \\
2 & 2 & 0 & 0 & 2 & 0
\end{array}\right) \text {. }
$$

Theorem 1 suggests to look at the distribution of the non-zero entries in the matrix. If we do so, we notice that there is indeed a square submatrix of $A_{1}$ of order $k=2$ with four, i.e., $2 k$, non-zero entries, namely

$$
D=\left(\begin{array}{ll}
2 & 2 \\
2 & 2
\end{array}\right)
$$

This is sufficient to conclude that $A_{1}$ is not irreducible, which is in line with [9]. Similarly, we can use Theorem 1 for Example 4 of [9]. Indeed, we consider the $(4 \times 6)$ matrix $A_{2}$ given as follows:

$$
A_{2}=\left(\begin{array}{llllll}
4 & 0 & 0 & 0 & 2 & 0 \\
0 & 2 & 0 & 2 & 2 & 0 \\
0 & 2 & 0 & 2 & 0 & 2 \\
0 & 0 & 4 & 0 & 0 & 2
\end{array}\right)
$$

We note that the $(2 \times 2)$ matrix $D$ is also a submatrix of $A_{2}$. Thus, $A_{2}$ is not an extreme point, which is again in accordance with [9].

We can use the same argument to conclude that the transportation matrix $T_{2}$ of Example 2 is not an extreme 
point. Indeed, there exists a $(2 \times 2)$ submatrix of $T_{2}$ with four positive entries, that is the following matrix:

$$
F=\left(\begin{array}{ll}
1 & 3 \\
2 & 2
\end{array}\right)
$$

We now discuss some cases of irreducible discretecopulas. Again, we start with an example originally presented in [9]. Namely, we look at the $(3 \times 8)$ matrix given as follows:

$$
A_{3}=\left(\begin{array}{llllllll}
3 & 3 & 0 & 0 & 0 & 2 & 0 & 0 \\
0 & 0 & 1 & 3 & 3 & 1 & 0 & 0 \\
0 & 0 & 2 & 0 & 0 & 0 & 3 & 3
\end{array}\right)
$$

If we analyze the matrix $A_{3}$, we notice that there are no submatrices of $A_{3}$ with the property that every row and column contains at least two positive entries. Therefore, $A_{3}$ is irreducible. From a similar argument we deduce that the matrix $T_{1}$ introduced in Example 1 is also an extreme point. In fact, the discrete copula associated with the matrix $T_{1}$ of Example 1 comes from the co-monotonic copula $M=\min \{u, v\}$.

Our last example for this section is a $(3 \times 8)$ matrix $A_{4}$ taken from [2] and defined as follows:

$$
A_{4}=\left(\begin{array}{llllllll}
3 & 3 & 2 & 0 & 0 & 0 & 0 & 0 \\
0 & 0 & 1 & 3 & 3 & 1 & 0 & 0 \\
0 & 0 & 0 & 0 & 0 & 2 & 3 & 3
\end{array}\right)
$$

As discussed in [2], the matrix $A_{4}$ is irreducible. Moreover, it is an example of an irreducible matrix that cannot be constructed extending a $(3 \times 6)$ extreme point of the corresponding generalized Birkhoff polytope. Therefore, it shows that the construction given in [16] fails to capture all the irreducible discrete copulas. We now have a closer look at the matrix $A_{4}$ by analyzing the first $(3 \times 6)$ matrix block:

$$
B_{4}=\left(\begin{array}{llllll}
3 & 3 & 2 & 0 & 0 & 0 \\
0 & 0 & 1 & 3 & 3 & 1 \\
0 & 0 & 0 & 0 & 0 & 2
\end{array}\right)
$$

We notice that $B_{4}$ cannot be associated to a discrete copula defined on $I_{3} \times I_{6}$ because its row sums are not uniform. Though, $B_{4}$ belongs to the transportation polytope $\mathscr{T}(\tilde{u}, \tilde{v})=\mathscr{T}([8,8,2],[3,3,3,3,3,3])$, which contains all transportation matrices corresponding to discrete copulas defined on the grid domain $U_{3} \times V_{6}=$ $\{0,1 / 6,1 / 3,1\} \times\{0,1 / 6,1 / 3,1 / 2,2 / 3,5 / 6,1\}$. By means of Theorem 1 , we derive that $B_{4}$ is an extreme point of $\mathscr{T}(\tilde{u}, \tilde{v})$. This suggests that the construction of irreducible discrete copulas given in [16] might work, in principle, if boundary cases like the one of matrix $A_{4}$ could also be included. However, alternative ways of constructing irreducible transportation matrices with uniform marginals can be found in [4].

\section{Conclusion}

In this paper, we provide a simple characterization of the (non-) extreme points of the space of arbitrary discrete copulas in terms of the zero patterns of their associated transportation matrices. We consider several examples and demonstrate how the presented characterization can be used to quickly check if a discrete copula is irreducible.

Although the focus of this work is on discrete copulas, a natural question arises whether or not similar characterizations can be obtained for discrete quasi-copulas. To the best of the authors' knowledge, there are no similar results for the alternating transportation polytopes, i.e., the convex polytopes that correspond to the space of discrete quasi-copulas. We know that the alternating transportation polytopes are much more complex than the transportation polytopes, and a generalization of the characterization of Theorem 1 is not obvious. Though, the alternating transportation polytopes appeared in the discrete geometry literature recently [11], and many properties of these polytopes have not been studied yet. For example, the complete description in terms of minimal bounding hyperplanes of the alternating transportation polytopes with uniform margins has been given in [18]. Still, there are many open questions regarding these polytopes which all correspond to interesting questions for the discrete quasi-copulas introduced in [18]. Such research questions deserve the attention of our research community and will be the focus of future work.

\section{Acknowledgement}

The authors would like to thank Juan Fernández Sánchez for reading an earlier version of the manuscript. The second author acknowledges the support by MIUR-PRIN 2017, Project "Stochastic Models for Complex Systems" (No. 2017JFFHSH).

\section{References}

[1] I. Aguiló, J. Suñer, J. Torrens, Matrix representation of discrete quasi-copulas, Fuzzy Sets and Systems 159 (13) (2008) 1658-1672.

[2] I. Aguiló, J. Suñer, J. Torrens, Matrix representation of copulas and quasi-copulas defined on nonsquare grids of the unit square, Fuzzy Sets and Systems 161 (2) (2010) 254-268.

[3] M. Bhaskara Rao, K. Subramanyam, The structure of some classes of bivariate distributions and some applications, Computational Statistics \& Data Analysis 10 (1990) 175 - 187. 
[4] R. M. Caron, X. Li, P. Mikusiński, H. Sherwood, M. D. Taylor, Nonsquare "doubly stochastic" matrices, in: L. Rüschendorf, B. Schweizer, M. Taylor (Eds.), Distributions with fixed marginals and related topics (Seattle, WA, 1993), Vol. 28 of IMS Lecture Notes Monogr. Ser., Inst. Math. Statist., Hayward, CA, 1996, pp. 65-75.

[5] E. de Amo, M. Díaz Carrillo, F. Durante, J. Fernández Sánchez, Extensions of subcopulas, J. Math. Anal. Appl. 452 (1) (2017) 1-15.

[6] J. A. De Loera, E. D. Kim, Combinatorics and geometry of transportation polytopes: An update, in: Discrete Geometry and Algebraic Combinatorics, Vol. 625 of Contemporary Mathematics, American Mathematical Society, Providence, RI, 2014, pp. 37-76.

[7] F. Durante, E. Perrone, Stochastic dependence with discrete copulas, in: A. Pollice, N. Salvati, F. Schirripa Spagnolo (Eds.), Book of Short Papers SIS 2020, Pearson, 2020, pp. 1344-1349.

[8] F. Durante, C. Sempi, Principles of Copula Theory, CRC/Chapman \& Hall, Boca Raton, FL, 2015.

[9] J. Fernández-Sánchez, J. J. Quesada-Molina, M. Úbeda Flores, New results on discrete copulas and quasi-copulas, Fuzzy Sets and Systems (in press).

[10] H. Joe, Dependence Modeling with Copulas, 2nd Edition, Chapman and Hall/CRC, Boca Raton, FL, 2014.

[11] V. Knight, Alternating sign matrices and polytopes, Ph.D. thesis (2009).

[12] A. Kolesárová, R. Mesiar, J. Mordelová, C. Sempi, Discrete Copulas, IEEE Transactions on Fuzzy Systems 14 (5) (2006) 698-705.

[13] V. Kuzmenko, R. Salam, S. Uryasev, Checkerboard copula defined by sums of random variables, Dependence Modeling 8 (1) (2020) 70 92.

[14] G. Mayor, J. Suñer, J. Torrens, Copula-like operations on finite settings, IEEE Transanctions on Fuzzy Systems 13 (4) (2005) 468-477.

[15] R. Mesiar, Discrete copulas-what they are, in: Proceedings of EUSFLAT- LFA Conference (Barcelona, Spain), 2005, pp. 927-930.

[16] J. Mordelová, A. Kolesárová, Some results on discrete copulas, in: Proceedings of the Fourth International Summer School on Aggregation operators (Ghent, Belgium), 2007, pp. 145-150.
[17] R. B. Nelsen, An Introduction to Copulas (Springer Series in Statistics), 2nd Edition, Springer, 2006.

[18] E. Perrone, L. Solus, C. Uhler, Geometry of discrete copulas, Journal of Multivariate Analysis 172 (2019) $162-179$.

[19] J. Piantadosi, P. Howlett, J. Boland, Matching the grade correlation coefficient using a copula with maximum disorder, Journal of Industrial \& Management Optimization 3 (1) (2007) 305-312.

[20] J. Piantadosi, P. Howlett, J. Borwein, Copulas with maximum entropy, Optimization Letters 6 (1) (2012) 99-125.

[21] J. J. Quesada Molina, C. Sempi, Discrete quasicopulas, Insurance: Mathematics and Economics 37 (1) (2005) 27-41.

[22] N. F. A. Radi, R. Zakaria, J. Piantadosi, J. Boland, W. Z. W. Zin, M. A.-z. Azman, Generating Synthetic Rainfall Total Using Multivariate Skew- $t$ and Checkerboard Copula of Maximum Entropy, Water Resources Management 31 (5) (2017) 1729-1744.

[23] L. Rüschendorf, On the distributional transform, Sklar's theorem, and the empirical copula process, Journal of Statistical Planning and Inference (2009) 3927-3927.

[24] J. Segers, Asymptotics of empirical copula processes under non-restrictive smoothness assumptions, Bernoulli 18 (3) (2012) 764-782.

[25] A. Sklar, Fonctions de répartition à $\mathrm{n}$ dimensions et leurs marges, Publications de l'Institut de Statistique de Paris 8 (1959) 229-231.

[26] J. Striker, The Alternating Sign Matrix Polytope, The Electronic Journal of Combinatorics 16 (1) (2009) R41.

[27] G. M. Ziegler, Lectures on Polytopes, SpringerVerlag, 1995. 